as a video of the webinar, can be found on the ADFM website at: http://www.adfammed.org/Members/Webinarsresources. On the website, the webinar reached far more than just the real-time participants, with over 150 "unique" visitors during the 2 weeks following the webinar, many of whom visited the site multiple times.

Participants' questions during the course of the presentations pertained to the following issues: (1) financial stability and sustainability; (2) students/ learners as "change agents" and the notion that they "eat this up"; (3) using real clinical sites for learning as opposed to simulation ${ }_{i}(4)$ taking advantage of opportunities in IPE for scholarship and telehealth/distance learning $_{i}(5)$ leadership development and teaching learners what it means to work as a team, not just to form teams $s_{i}(6)$ addressing professional biases about hierarchy on teams; (7) logistical challenges of scheduling with physical distances between learners' professional schools (one area where distance learning can be very helpful); and (8) tools for assessing teamwork.

Dr. Rodgers summarized several important points for everyone moving towards IPE within their own institutions. First, the biggest issue confronting IPE is financial support and sustainability, as creating a stable and effective program is resource intensive. Grants are an important source of funding, but we need to consider whether programs can be sustainable when grant funds are gone-and need to figure out ways to bring in IPE innovations that are cost- and time-effective.

Second, ensure that IPE is seen as critical in a number of clinical settings, not just ambulatory care. Including IPE in the inpatient setting is extremely important, although it may be the most difficult to carry out.

Third, there are research questions we should be addressing in our work around IPE. How much IPE is enough? At what point do we introduce IPE so that students' appreciation for working as members of a team is maintained throughout their clinical careers? How will we measure whether or not our interventions on the educational side mattered? How do we overcome the logistical challenges? Which IPE experiences are best?

Finally, Dr. Rodgers noted that moving students into hospital settings where they see less-than-ideal examples of interprofessional communication and collaboration undermines the education received on the importance of working in interprofessional teams and how to be collaborative across professions. Although we are beginning to see some evidence that the introduction of IPE into an institution may actually cause the faculty and the clinicians to look at how well they are collaborating interprofessionally in their own clinical practices, a larger question remains about how raising student expectations about team performance may influence those who are teaching them to perform better as members of teams. More broadly, this also raises the issue of the integration of interprofessional practice, both its importance and the "how." ADFM plans to hold a follow-up webinar in the spring focusing on the issues around interprofessional practice.

Ardis Davis, Amanda Weidner, Denise Rodgers, Alfred Tallia, Chris Matson, \& members of ADFM's Healthcare Delivery and Education Transformation Committees

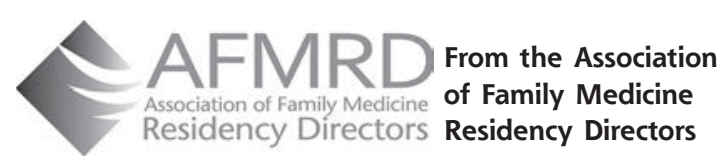

Ann Fam Med 2015;13:91-92. doi: 10.1370/afm.1744

\section{TRAINING IMPLICATIONS OF FAMILY MEDICINE FOR AMERICA'S HEALTH: A PREVIEW}

Family Medicine for America's Health was launched in October 2014, heralding a major initiative by family medicine organizations across the country to reform health care. This initiative is focused on 6 key implementation areas: practice, payment, workforce education and development, technology, research, and engagement. Family medicine program directors will serve an essential role in workforce education and development.

The primary care workforce shortage has been recognized as one of the major deficiencies in our health system. As program directors, we have a responsibility to not only educate more family physicians, but to ensure our graduates are prepared for a different health care system. If we are to produce a workforce of family physicians who will thrive in a new environment and deliver higher quality care with greater value, many aspects of medical school, residency, and CME demand some redesign.

Considering the prominent roles that family physicians will serve within tomorrow's medical system, our graduates must be equipped with an increasingly wider range of skills and characteristics, including the following:

1. Diagnostic: Family physicians will be expected to have a broad medical knowledge base across specialty domains and patient assessment skills for undifferentiated patients of all ages. This is a core skill of critical importance and the key to providing the right care at the right time for all patients in the practice. 
2. Acute care: We must be able to diagnose acute conditions ranging from minor to severe illness and manage their initial treatment.

3. Chronic disease management: Family physicians will require a mastery of chronic disease care for the most common diseases in the population as well as prevention and early recognition of complications.

4. Secondary (hospital) care: Family physicians must have the ability to transition care to and from outpatient and inpatient settings, coordinating care by either managing inpatient care directly or closely working with hospitalists to assure care is personalized and efficient.

5. Maternity care: The level of maternity care that family physicians will provide will be based on the community they are serving. Residency training will include the skill sets outlined by the CAFM Maternity Care Training Guidelines that will be published this spring.

6. Primary care team leadership: It is essential that family physicians have training in leadership skills, team-based care, quality improvement and safety. By the nature of their skill sets, family physicians will frequently be relied upon to direct the care team in the management of the practice's population.

7. Patient advocacy: Our graduates must understand the sociology of health. The profound impact of socioeconomic factors on the patient, the family, and the community is well documented and must be at the forefront of our advocacy work as physicians within our communities.

8. Mental health partnering: Because family physicians evaluate and manage a high percentage of mental illness within our medical system, our training in primary interventions, treatment, and integration of mental health services within the primary care clinic will be crucial.

9. Health systems management: Because of the breath of our practice and knowledge of the community, family physicians will play a critical role in the design of future health systems.

Medical education is a continuum. Program directors cannot ignore the training in medical school nor can we neglect the education of practicing physicians as we move our specialty forward.

There is much to do in medical schools across our country to increase the pipeline of students entering family medicine and to prepare them to enter residency. Continuing medical education for practicing family physicians will need to address the core expectations of family physicians outlined above. There will be a need for retraining and re-expansion of the scope of practice for some of our physicians as we take on more responsibility for the care of our populations.

The incentives will change significantly as we expand the role of primary care and encourage physicians to employ a variety of skills in delivering care to the patient. In the new model of care that is envisioned, we will be asked to provide care of greater value and not of greater volume. In the end, the greatest value we can bring is the robust set of skills outlined here into the clinics we work in every day.

Michael Tuggy, MD

Natasha Bbuyan, $M D_{i}$ Gretchen M. Dickson, $M D, M B A_{i}$ James W. Jarvis, $M D_{i}$ Lisa Maxwell, $M D_{i}$ Michael Mazzone, $M D_{i}$ W. Fred Miser, $M D_{i}$ Karen Mitchell, MD, Stephen Scbultz, MDi Todd Shaffer, MD, MBA 